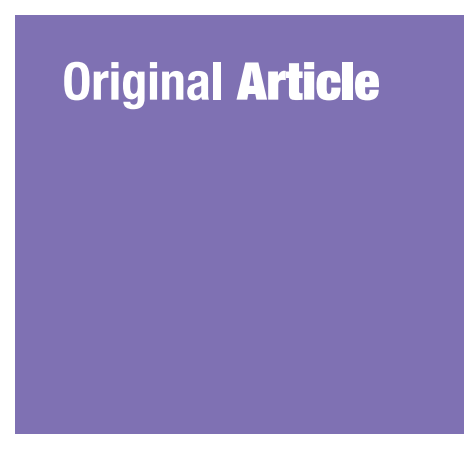

Submitted: 23 Dec 2020 Accepted: 10 Apr 2021 Online: 26 Aug 2021

\section{Comparing the Therapeutic Effects of Crocin, Escitalopram and Co-Administration of Escitalopram and Crocin on Learning and Memory in Rats with Stress-Induced Depression}

\author{
Mehran Joodakı, Maryam Radahmadi, Hojjatallah AlaeI
}

Department of Physiology, School of Medicine, Isfahan University of Medical Sciences, Isfahan, Iran

To cite this article: Joodaki M, Radahmadi M, Alaei H. Comparing the therapeutic effects of crocin, escitalopram and co-administration of escitalopram and crocin on learning and memory in rats with stress-induced depression. Malays J Med Sci. 2021;28(4):50-62. https://doi.org/10.21315/mjms2021.28.4.6

To link to this article: https://doi.org/10.21315/mjms2021.28.4.6

\begin{abstract}
Background: Depression affects various brain functions. According to previous studies, escitalopram influences brain functions in depression and crocin reduces memory impairments. Therefore, this study aimed to compare the therapeutic effects of using crocin and escitalopram (separately and in combination) on learning and memory in rats with stress-induced depression.

Methods: Fifty-six rats were allocated into seven groups of control, sham, continuous depression, recovery period, daily injections of escitalopram, crocin and escitalopram-crocin during 14 days after inducing depression by stress. Passive avoidance (PA) test was used to assess brain functions.

Results: Latency has significant differences in depression group. Also, it significantly increased in depression-crocin, depression-escitalopram and depression-escitalopram-crocin groups compared to the depression group. The dark stay (DS) time was significantly higher in the depression and depression-recovery groups. However, the DS time significantly decreased in the depression-crocin, depression-escitalopram and depression-escitalopram-crocin groups. Furthermore, the number of entrances to the dark room was significantly lower in depressioncrocin and depression-escitalopram-crocin groups compared to the depression one.

Conclusion: Different depression treatments (i.e. crocin, escitalopram and crocinescitalopram) reduced depression-induced memory deficits. Crocin and escitalopram-crocin, respectively, improved brain functions and locomotor activity more than escitalopram. Comparatively, in subjects with depression, crocin, which is an effective saffron constituent, partially affected the memory deficits better than escitalopram (as a chemical component).
\end{abstract}

Keywords: depression, stress, memory, learning, crocin, escitalopram

\section{Introduction}

Depression is a heterogeneous disease triggered by social, biological, genetic and psychological (e.g. stressful life events) factors $(1,2)$. It affects many brain functions like cognition, memory, moods and other behaviours involving the limbic-cortical networks (3-6).
This condition changes some neurobiological factors, including neurotransmitters (particularly serotonin), hormones, as well as inflammatory and neurotrophic factors in the brains of subjects with depression $(5,7-10)$. Nowadays, escitalopram or other selective serotonin reuptake inhibitors (SSRIs) are the first-line depression treatments (11). Nevertheless, the 
role of SSRIs on cognitive performance is not thoroughly clarified yet. The positive effects of SSRI drugs on cognitive functions among subjects with depression are previously reported in some studies $(5,11-13)$. Conversely, some research studies have indicated no improvement in memory due to using these drugs $(14,15)$.

Notably, some herbal drugs, such as saffron (Crocus sativus) play a definitive role in brain functions and cognitive capacity (16). Crocin is the most biologically active component of saffron (17) that can improve cognitive abilities, memory, anxiety and depression $(17,18)$. It can also improve the functionality of the central nervous system by regulating the serotonin synthesis (19). Even though both escitalopram and crocin affect serotonin secretion, they could probably have different mechanisms in affecting cognitive functions efficiently. Previous studies have focused on the effect of either crocin or escitalopram on providing treatment for depression; but no published report is yet available about the comparative therapeutic effects of crocin, escitalopram, co-administration of escitalopram and crocin, as well as the impact of allowing a recovery period after depression on cognitive functions. Therefore, this study aims to investigate which treatment (i.e. crocin, escitalopram, co-administration of escitalopram and crocin or recovery period after depression) would impact learning, memory, memory consolidation and locomotor activity in subjects with stress-induced depression (using behavioural tests).

\section{Methods}

\section{Animals}

This experiment was performed using 56 adult male Wistar rats (with initial weights of $200 \mathrm{~g}-250 \mathrm{~g}$ ). These rats were obtained from Isfahan University of Medical Sciences and were housed under controlled environmental conditions $\left(23 \pm 2{ }^{\circ} \mathrm{C}\right.$ temperature and humidity of $50 \% \pm 5 \%$ (mean \pm standard deviation [SD]) with a 12:12 light/dark cycle (lights on from 07:00 to 19:00). Similar food and water were made accessible ad libitum except during the stress induction phase. Each colony group consisted of four rats in a cage. The experiment period lasted for 28 days. All behavioural tests were conducted between 14:00 and 16:00 daily. The Ethics Committee of Animal Use at the Isfahan University of Medical Sciences approved the study. Hence, all experiments were conducted in compliance with the Guide for the Care and Use of Laboratory Animals in Iran. After the adaptation of animals for a week, they were randomly divided into the following seven groups $(n=8)$ :

(i) Control (Co) group: Rats were handled like the ones in the experimental groups during the study period and received no special treatments.

(ii) Sham (Sh) group: Rats received equal volumes of normal saline (drug vehicle) consecutively for the next 14 days.

(iii) Depression-recovery (Dep-Rec) group: Rats were under restraint stress (6 h daily for 14 days); subsequently, they were incaged for the next 14 days (recovery period).

(iv) Depression-depression (Dep-Dep) group: Rats were under restraint stress $(6 \mathrm{~h}$ daily, for 14 days) during two subsequent periods of the next 14 days (overall, 28 days).

(v) Depression-crocin (Dep-Cr) group: Rats were under chronic restraint stress $(6 \mathrm{~h}$ daily, for 14 days); then they received daily injections of $30 \mathrm{mg} / \mathrm{kg}$ crocin for the next 14 days.

(vi) Depression-escitalopram (Dep-Esc) group: Rats were under chronic restraint stress (6 h daily, for 14 days); then they received daily injections of $20 \mathrm{mg} / \mathrm{kg}$ escitalopram for the next 14 days.

(vii) Depression-escitalopram-crocin (DepEsc-Cr) group: Rats were under chronic restraint stress ( $6 \mathrm{~h}$ daily, for 14 days); then they received daily injections of $20 \mathrm{mg} / \mathrm{kg}$ escitalopram and $30 \mathrm{mg} / \mathrm{kg}$ crocin for the next 14 days (Figure 1).

\section{Experimental Procedures}

\section{Depression paradigm}

To induce depression, rats were placed in Plexiglas cylindrical restrainers for $6 \mathrm{~h}$ daily (8:00-14:00) during 14 days (20-22). On day 14, after verifying the induction of depression by a forced swim test (FST), the the rat with depression were introduced to the experimental protocol. An increase in immobility was seen as the induction of depression (23). 


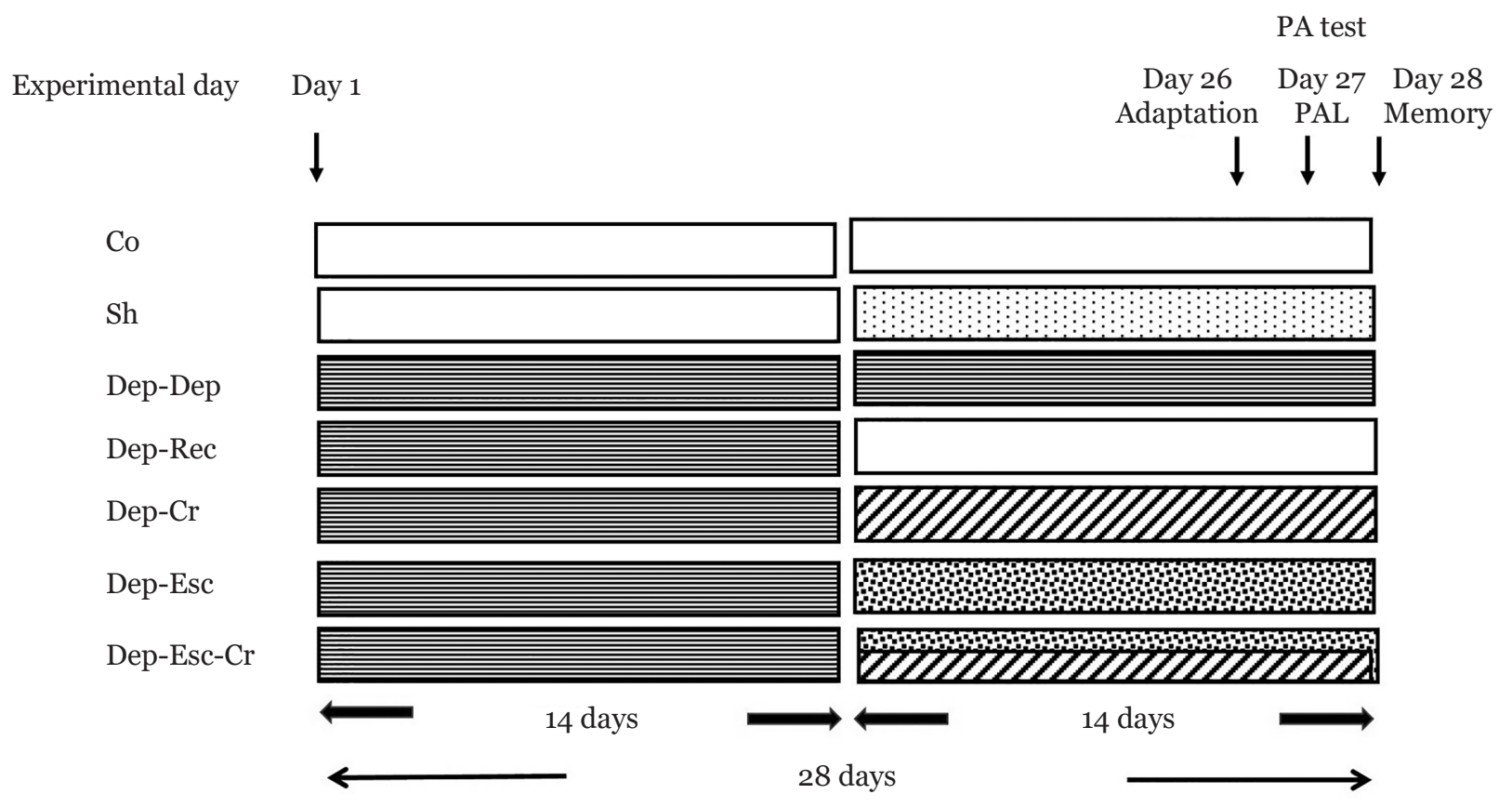

Figure 1. The schematic diagram of different groups

\section{Chemicals and reagents}

The subjects with depression received intraperitoneal injections of escitalopram (20 mg/kg; Sobhan Darou Pharmaceutical Co., Iran) and crocin (30 mg/kg; Sigma-Aldrich Co., USA), dissolved in normal saline for 14 consecutive days.

\section{Behavioural paradigms}

To assess learning, memory, memory consolidation and locomotor activity involving cognitive memory, the passive avoidance (PA) test was used. The PA apparatus (Shuttle-box, $64 \mathrm{~cm} \times 25 \mathrm{~cm} \times 35 \mathrm{~cm}$ ) was divided into two identical compartments $(32 \mathrm{~cm} \times 25 \mathrm{~cm} \times 35 \mathrm{~cm})$ with a sliding door and grid floors. After a 300sec habituation period in the apparatus (day 26), a single learning trial was performed (day 27) by delivering an electric shock through the grid floor $(0.5 \mathrm{~mA}, 50 \mathrm{v}$ and $2 \mathrm{sec})$ to the animal's foot using an isolated stimulator. Subsequently, on day 28, the PA memory trial was conducted. Further details of the PA test were based on our previously published reports $(18,24)$. The initial latency time to enter the dark compartment was recorded before inducing the electric shock. In the memory trial on day 28 , the latency to enter the dark room was measured up to a maximum delay of 300 sec. If the rat did not enter the dark compartment within $300 \mathrm{sec}$, the memory trial was terminated.
The difference between the initial latency and the latency after one day was interpreted as the occurrence of learning (25). Moreover, the latency of entrance to the dark compartment after one day was considered for measuring the short-term memory (26). The total dark stay (DS) time was assigned as either memory consolidation and/or storage of new information (25). Similarly, the number of entrances to the dark compartment was recorded as the locomotor activity $(27,28)$. Furthermore, the animal's ability to remember the received foot shock was determined by the PA test. Avoidance to enter the dark compartment and even longer stay periods in the light compartment were interpreted as a positive response in the trial (25).

\section{Data analysis}

All data were estimated as mean \pm standard error of mean (SEM) and analysed by analysis of variance (ANOVA) test, followed by the Least Significant Difference (LSD) post-hoc testing for multiple groups. The paired-sample $t$-test was used to compare the initial latency and latency after one day (within-group). Additionally, the $P$-value less than 0.05 was considered statistically significant. All calculations were performed using IBM SPSS Statistics v.24. 


\section{Results}

In the current study, the Co and Sh groups exhibited no significant difference in the behavioural test. Therefore, the Co group was selected as a reference for all following comparisons.

\section{Assessment of Learning and Memory Functions}

As illustrated in Figure 2, no significant differences were observed in initial latency values among all groups. The latency after one day was significantly $(P<0.01)$ lower in the Dep-Dep group compared to the Co group; this indicated the induction of memory impairment by depression (Figure 3). Also, there was no significant difference in the latency after one day between the Dep-Rec and Co groups (Figure 3). However, the latency showed significant enhancements in the Dep-Cr and Dep-Esc-Cr groups $(P<0.01$ for both) and the Dep-Esc group $(P<0.05)$ compared to the DepDep group. Therefore, the beneficial effects of crocin and escitalopram treatments on reducing the depression-induced memory deficit was indicated (Figure 3).
The initial latency and latency after one day were analysed using a paired-sample $t$-test to evaluate within-group latency changes. Significant differences were detected between the initial latency and latency after one day in all groups $(P<0.001$, except for the Dep-Dep group $(P<0.05)$, indicating the occurrence of learning in all experimental groups (Figure 4).

In both Dep-Rec and Dep-Dep groups, the DS time was significantly $(P<0.01)$ higher than Co group, indicating the impairment of memory consolidation in depression with and without a recovery period. However, the total DS time had significant decreases in the Dep-Cr, Dep-Esc-Cr groups $(P<0.01)$ and Dep-Esc group $(P<0.05)$ compared to the Dep-Dep group (Figure 5).

The number of entrances to the dark compartment showed no significant difference among the Dep-Rec, Dep-Dep and Dep-Esc groups ( $P>0.05$ for all groups) compared to the Co group (Figure 6).

However, there were significant decreases in the Dep-Cr group compared to the Co and Dep-Rec groups, respectively $(P<0.01$ and $P<$ 0.05). Similar enhancements were also observed in the Dep-Esc-Cr group compared to the Co and Dep-Rec groups, respectively $(P<0.01$ and $P<0.05)$, indicating a reduced locomotor activity in both groups due to the use of crocin treatment in depression (Figure 6).

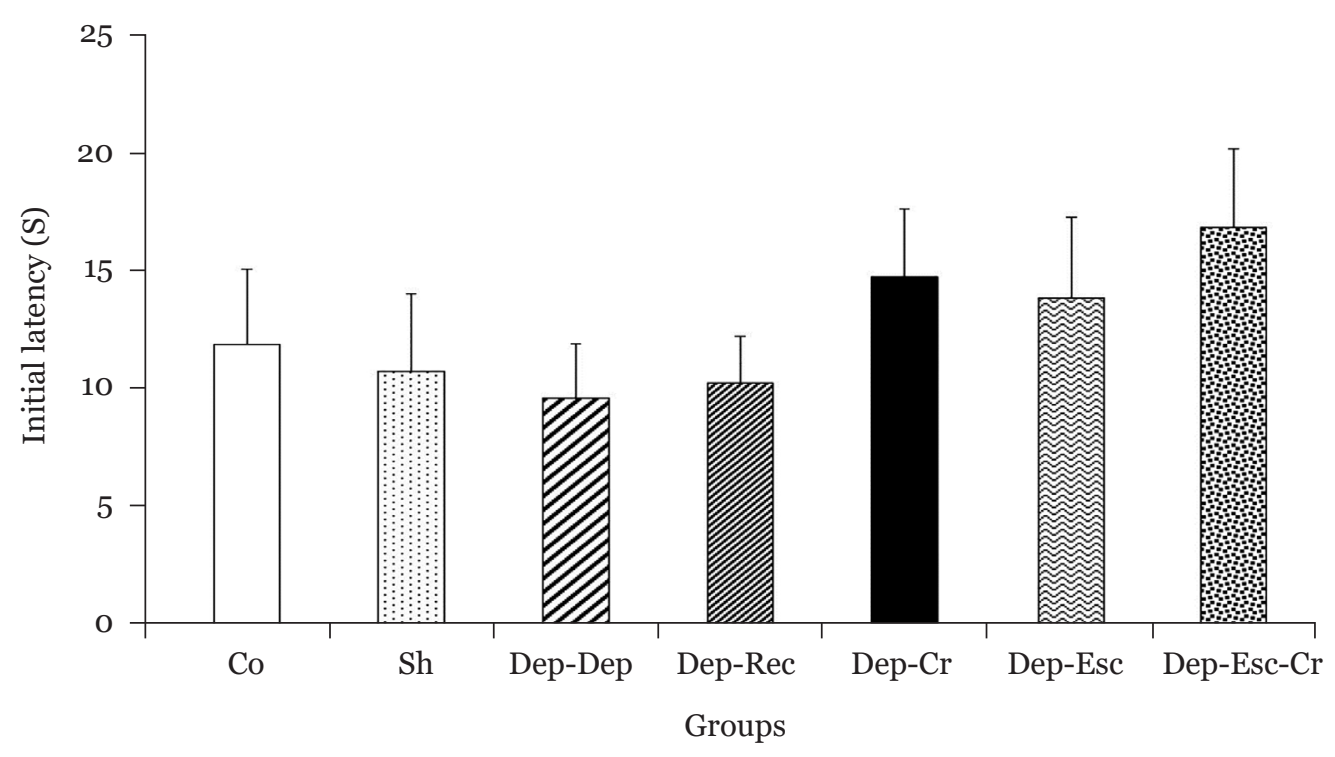

Figure 2. The initial latency to enter the dark room (of the PA apparatus) before receiving foot-shock for all groups $(n=8)$. Results are expressed as mean \pm SEM. No significant differences were observed among all groups 


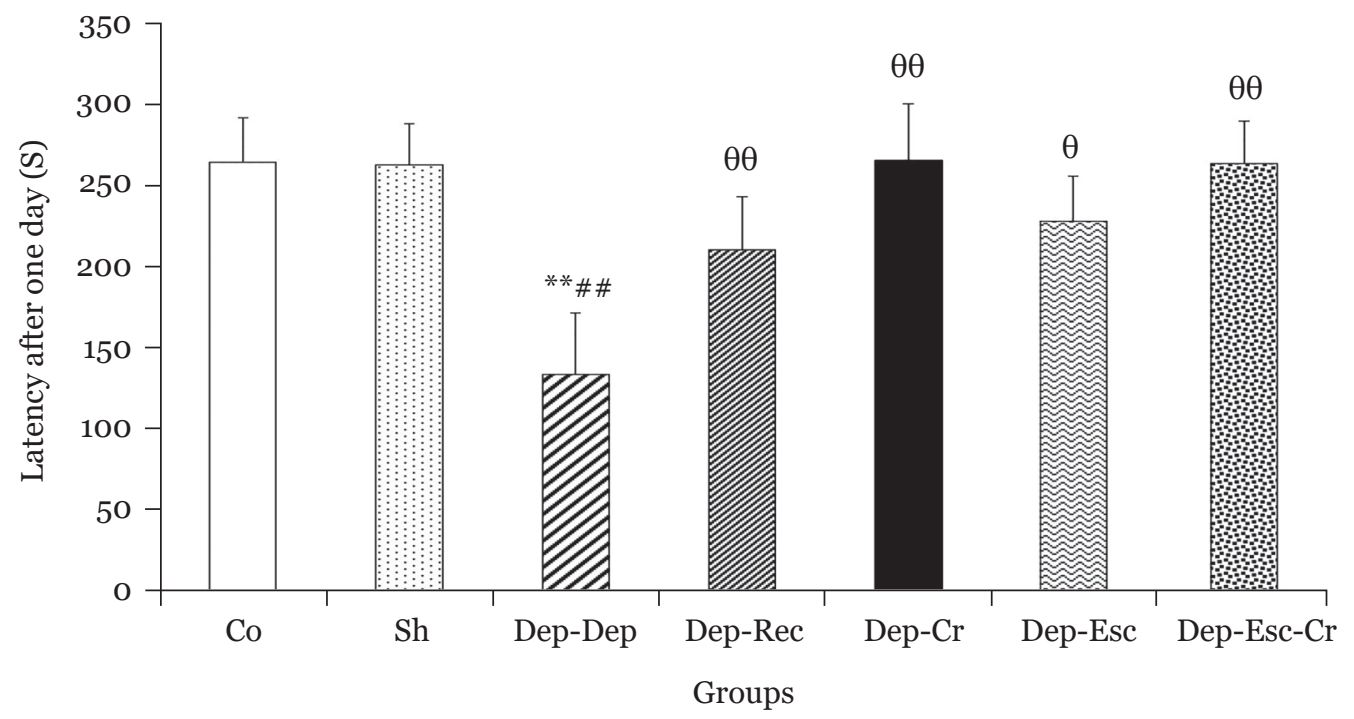

Figure 3. Latency to enter the dark room (of the PA apparatus), one day after receiving the foot shock for all groups $(n=8)$. Results are expressed as mean \pm SEM

Notes: ${ }^{* *} P<0.01$ compared to the Co group; ${ }^{\# \# P} P$ 0.01 compared to the Sh group; ${ }^{\theta} P<0.05$ and ${ }^{\theta \theta} P<0.01$ compared to the Dep-Dep group

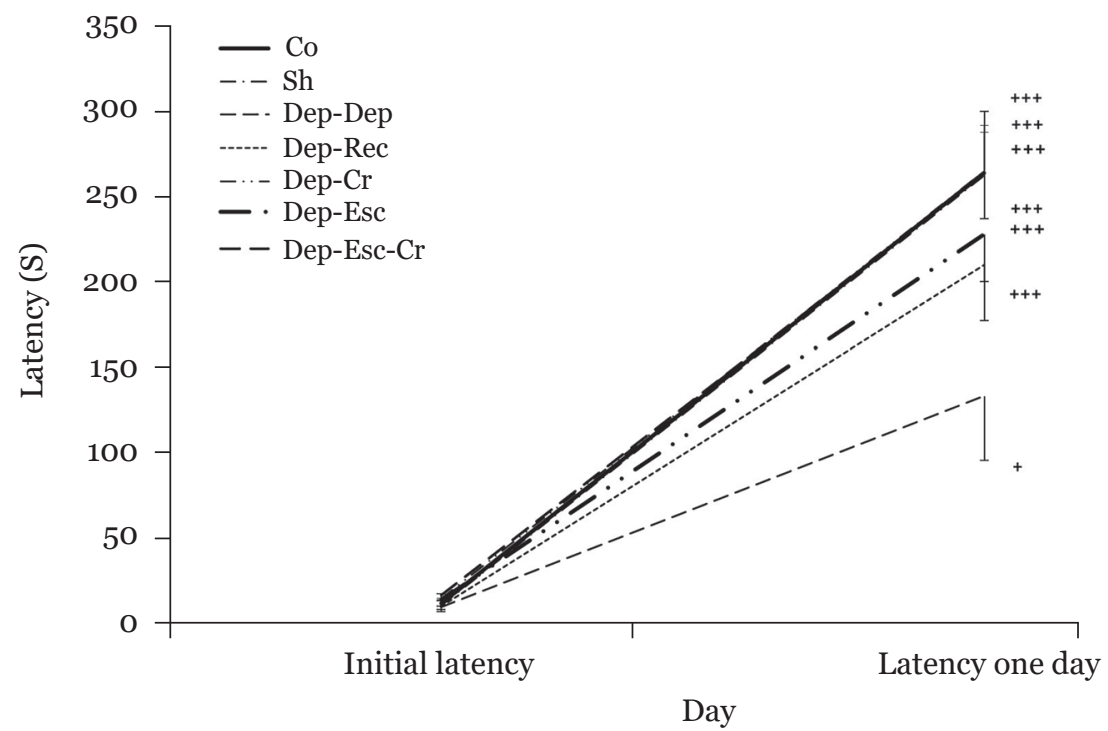

Figure 4. Initial latency and latency after one day to enter the dark room (of the PA apparatus), respectively, before and after the foot shock (within-group) $(n=8)$. Results are expressed as mean \pm SEM

Notes: ${ }^{+} P<0.05$ and ${ }^{++} P<0.001$ Initial latency compared to latency after one day

\section{Discussion}

In this study, a comparison was conducted between the therapeutic effects of crocin, escitalopram, co-administration of escitalopram and crocin, as well as the recovery period after depression on cognitive functions (e.g. learning, memory, memory consolidation and locomotor activity) in rats with stress-induced depression.

Current findings revealed that learning occurred in all experimental groups although depression diminished the level of learning. As such, different treatments, such as the recovery period, crocin, escitalopram and escitalopram- 
Original Article | Escitalopram-crocin co-administration on memory

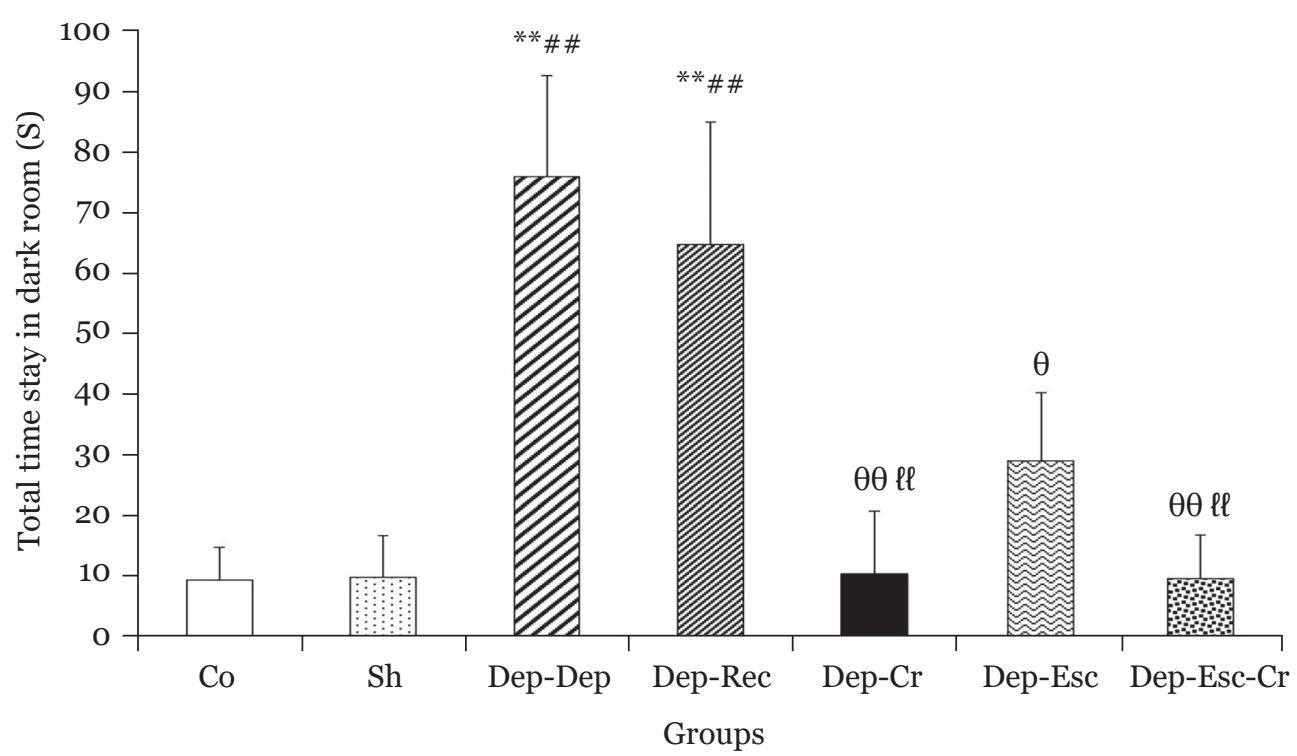

Figure 5. Total stay time in the dark room (of the PA apparatus), one day after receiving the foot-shock for all groups $(n=8)$. Results are expressed as mean \pm SEM

Notes: ${ }^{* *} P<0.01$ compared to the Co group; ${ }^{\# \#} P<0.01$ compared to the Sh group; ${ }^{\theta} P<0.05$ and ${ }^{\theta \theta} P<0.01$ compared to the Dep-Dep group; and finally, ${ }^{\text {lP}} P<0.01$ compared to the Dep-Rec group

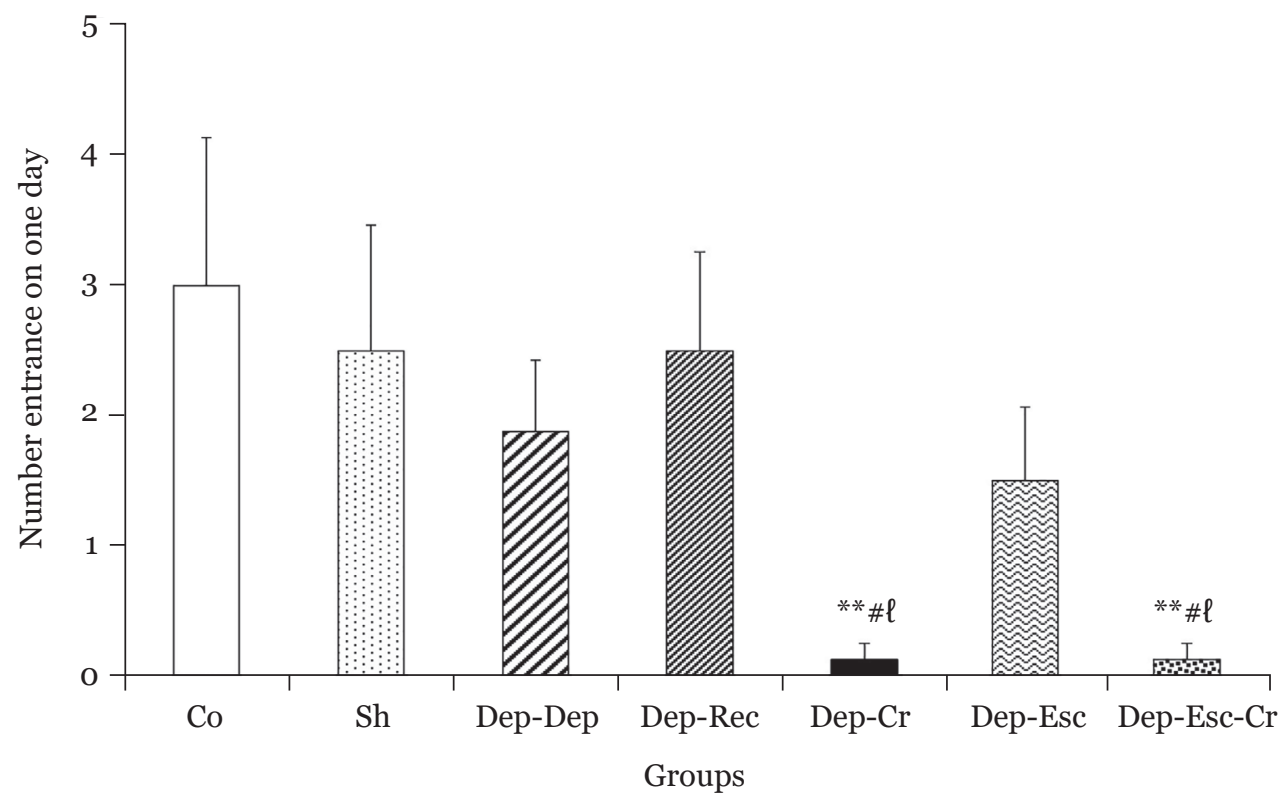

Figure 6. The number of entrances to the dark room in the PA apparatus for all groups one day after receiving the foot shock $(n=8)$. Results are expressed as mean \pm SEM

Notes: ${ }^{* *} P<0.01$ compared to the Co group, ${ }^{*} P<0.05$ compared to the Sh group and ${ }^{\ell} P<0.05$ compared to the Dep-Rec group

crocin improved learning slightly among depression groups. According to some studies, depression often causes learning impairments (29, 30). Accordingly, Gao et al. (31) showed that learning decreased under the chronic unpredictable stress-induced depression models. Also, some reports have demonstrated that some materials, such as escitalopram and crocin may revert the learning impairments (32-36). Contrastingly, Skandali et al. (37) demonstrated 
that escitalopram not only did not improve learning, but also led to learning impairment among healthy volunteers.

In the present study, memory and memory consolidation were impaired by induction of depression. Consistent with these findings, depression accelerated the severity of cognitive impairments in other research studies $(38-40)$. As Harmer et al. (41) suggested, the decrease in serotonin levels among depressed subjects caused impairment of memory consolidation.

Another important finding of the present study is that although the recovery period after depression slightly improved memory, it was not adequate to revert memory impairment among depressed subjects. Moreover, memory consolidation was severely destructed in subjects with depression (even when there was a recovery period).

According to other findings, different treatments, including the use of escitalopram, and particularly, crocin and crocin-escitalopram co-administration, improved memory consolidation and reduced depression-induced memory deficit. Moreover, the effects of escitalopram on other types of memory $(12,32)$ or the impact of citalopram (an older version of escitalopram) on memory consolidation are reported (41). However, Jensen et al. (42) have indicated that escitalopram did not revert those memory impairments that were induced by central 5-HT depletion (42). Also, in another study, escitalopram did not affect working memory (43). These contradictory results may have been due to the type of induced depression, strain, gender, drug dose and behavioural test (44). Nevertheless, there are pieces of evidence of memory improvement by crocin under various conditions (25, 45-47). Accordingly, Heidari et al. (48) showed that crocin could have a preventive effect on the memory impairments that were caused by age-related brain disorders, such as Alzheimer's disease. Additionally, the beneficial impact of crocin on streptozotocininduced memory impairment was seen to be related to antioxidant activities (49).

Interestingly, based on this study, even though using escitalopram and crocin separately improved memory impairments, the therapeutic effect of crocin, as an effective constituent of a medical plant, in the depression-induced memory deficits was stronger than the impact of escitalopram, which is a chemical component. Furthermore, the co-administration of crocin and escitalopram exerted more efficacy for the betterment of memory deficit in depression. As highlighted in a previous study, chronic stress would lead to the impairment of memory consolidation; whereas administration of crocin has improved it (25). Additionally, SSRIs have improved memory consolidation possibly due to the increase in serotonin levels (41). Different mechanisms were suggested for these beneficial effects of crocin and escitalopram on cognitive functions, namely: alteration of biochemical substances, brain neurotransmitters, hormones and the morphological changes in specific brain areas $(32,50-52)$. Both crocin and escitalopram are proven to have many similar beneficial effects. Nevertheless, the underlying mechanism of these effects with respect to providing treatments for memory impairments is not properly understood yet. For instance, it is demonstrated that both of these agents have antioxidant effects; hence, they both remove free radicals as one of the depression factors (53-55). Additionally, they prevent the elimination of neural cells by suppressing the effects of tumour necrosis factor (TNF) on neurons $(56,57)$. Moreover, they both increase serotonin in some brain regions $(12,19,25)$ and reduce the hyperactivity of the hypothalamicpituitary-adrenal (HPA) axis, which affects the hippocampal function and structure (58, 59). Therefore, further research is necessary to understand the differences between crocin and escitalopram. On one hand, it is proven that crocin increased hippocampal brain derived neurotrophic factor (BDNF) mRNA levels similar to some antidepressant drugs (17). On the other hand, in other studies, the antidepressant effects of escitalopram were unrelated to the regulation of hippocampal BDNF expression and serum BDNF levels among rats with depression (60, 61).

Other present findings revealed that depression decreased locomotor activity nonsignificantly in the PA test. However, Yang et al. (62) indicated that four weeks of unpredictable chronic stress (as a depression model) reduced locomotor activity in rats. Moreover, a decreased locomotor activity was observed by stressinduced depression using the open field test (63). By contrast, different evidence marked an increase in locomotor activity among mice with depression (64). Therefore, all factors, such as the type of behavioural test, rodent and stress, as well as duration and timing of stress-induced depression are important in various locomotor activity responses (62-64). 
Finally, the use of crocin and coadministration of escitalopram and crocin, but not escitalopram alone, severely decreased locomotor activity compared to the normal conditions with a recovery period after the depression. It seems that crocin, but not escitalopram, had an effective role in reducing locomotor activity. Consistently with the present findings, Hosseinzadeh et al. (65) indicated that locomotor activity was reduced by crocin in rodents. Contrastingly, via an open field test, locomotor activity was not affected by crocin (66). There are limited studies on the effects of escitalopram on locomotor activity in rats with depression. Despite these findings, the level of locomotor activity in rats decreased in the poststroke depression model while escitalopram reversed it (67). Also, Prinssen et al. (68) demonstrated an increase in locomotor activity by escitalopram. Thus, as previously mentioned, the level of responsiveness to locomotor activity may differ depending on the type of behavioural test and/or experimental protocol.

\section{Conclusion}

All in all, different treatments for depression, including the use of escitalopram, crocin, and co-administration of escitalopram and crocin reduced the depression-induced memory deficits. Respectively, crocin and coadministration of escitalopram-crocin acted better than escitalopram alone on improving the brain functions and locomotor activity in depression. Concerning the alleviation of depression-induced memory deficits, crocin (as an effective constituent of saffron) seems to have partially acted better than escitalopram which is a chemical component. However, further studies on other molecular, cellular, structural and biochemical mechanisms are needed to assess the effects of escitalopram and crocin on improving brain dysfunctions both separately or in combination.

\section{Acknowledgements}

The present research was made possible through the supports received from Isfahan University of Medical Sciences, Isfahan, Iran.

\section{Ethics of Study}

All procedures of this study were performed following the Guidelines for Care and Use of Laboratory Animals and National Institutes of Health (NIH Publications, No. 80-23, revised 2011) and approved by the Institute of Animal Care and Use Committee of Isfahan University of Medical Sciences (Ethics code: IR.MUI.MED. REC.1398.606).

\section{Conflict of Interest}

None.

\section{Funds}

This study would not have become possible without the financial support provided by Isfahan University of Medical Sciences, Isfahan, Iran.

\section{Authors' Contributors}

Conception and design: MR

Analysis and interpretation of the data: MR

Drafting of the article: MJ, MR, HA

Critical revision of the article for important

intellectual content: MR, HA

Final approval of the article: MR, HA

Obtaining of funding:MJ

Administrative, technical or logistic support: MR

Collection and assembly of data: MJ

\section{Correspondence}

Dr Maryam Radahmadi

$\mathrm{PhD}$ of Medical Physiology

(Isfahan University of Medical Sciences)

Department of Physiology,

School of Medicine, Isfahan University of

Medical Sciences, Isfahan, Iran.

Tel: 009803137929176

Fax: 009803136688597

E-mails:m_radahmadi@med.mui.ac.ir/ m_radahmadi@yahoo.com 


\section{References}

1. Boas GRV, de Lacerda RB, Paes MM, Gubert P, da Cruz Almeida WL, Rescia VC, et al. Molecular aspects of depression: a review from neurobiology to treatment. Eur J Pharmacol. 2019;851:99-121. https://doi.org/10.1016/j.ejphar.2019.02.024

2. Krishnan V, Nestler EJ. The molecular neurobiology of depression. Nature. 2008; 455(7215):894-902. https://doi.org/10.1038/ nature07455

3. Dudek KA, Dion-Albert L, Kaufmann FN, Tuck E, Lebel M, Menard C. Neurobiology of resilience in depression: immune and vascular insights from human and animal studies. Eur $J$ Neurosci. 2019;53(1):183-221. https://doi.org/ 10.1111/ejn.14547

4. Dillon DG, Pizzagalli DA. Mechanisms of memory disruption in depression. Trends Neurosci. 2018;41(3):137-149. https://doi.org/10.1016/ j.tins.2017.12.006

5. Dean J, Keshavan M. The neurobiology of depression: an integrated view. Asian J Psychiatr. 2017;27:101-111. https://doi.org/10.1016/j.ajp .2017 .01 .025

6. Frodl T, Meisenzahl EM, Zetzsche T, Born C, Groll C, Jäger M, et al. Hippocampal changes in patients with a first episode of major depression. Am J Psychiatry. 2002;159(7):1112-1118. https://doi.org/10.1176/appi.ajp.159.7.1112

7. Jain N, Steffens DC. Neurobiology and risk factors of late-life depression. Understanding depression. 2018;279-295. https://doi.org/10.1007/978-981 $-10-6580-4 \_23$

8. Vogelzangs N, Duivis HE, Beekman AT, Kluft C, Neuteboom J, Hoogendijk W, et al. Association of depressive disorders, depression characteristics and antidepressant medication with inflammation. Transl Psychiatry. 2012;2(2): e79-e88. https://doi.org/10.1038/tp.2012.8

9. Palazidou E. The neurobiology of depression. Br Med Bull. 2012;101(1):127-145. https://doi .org/10.1093/bmb/ldsoo4
10. Brummelte S, Galea LA. Depression during pregnancy and postpartum: contribution of stress and ovarian hormones. Prog Neuropsychopharmacol Biol Psychiatry. 2010;34(5):766-776. https://doi.org/10.1016/ j.pnpbp.2009.09.006

11. Waugh J, Goa KL. Escitalopram. CNS drugs. 2003;17(5):343-362. https://doi.org/10.2165/ 00023210-200317050-00004

12. do Couto FS, Batalha VL, Valadas JS, Data-Franca J, Ribeiro JA, Lopes LV. Escitalopram improves memory deficits induced by maternal separation in the rat. Eur $J$ Pharmacol. 2012;695(1-3): 71-75. https://doi.org/10.1016/j.ejphar.2012.08 .020

13. Wroolie TE, Williams KE, Keller J, Zappert LN, Shelton SD, Kenna HA, et al. Mood and neuropsychological changes in women with midlife depression treated with escitalopram. $J$ Clin Psychopharmacol. 2006;26(4):361-366. https://doi.org/10.1097/o1.jcp.oooo227699.26375 .f8

14. Kalechstein A, Mahoney III J, Yoon J, Bennett R, De La Garza II R. Modafinil, but not escitalopram, improves working memory and sustained attention in long-term, high-dose cocaine users. Neuropharmacology. 2013;64:472-478. https://doi.org/10.1016/j.neuropharm.2012.06.064

15. Yilmaz N, Demirdas A, Yilmaz M, Sutcu R, Kirbas A, Cure MC, et al. Effects of venlafaxine and escitalopram treatments on NMDA receptors in the rat depression model. $J$ Membr Biol. 2011;242(3):145-151. https://doi.org/10.1007/ soo232-011-9385-3

16. Gómez-Pinilla F. Brain foods: the effects of nutrients on brain function. Nat Rev Neurosci. 2008;9(7):568-578. https://doi.org/10.1038/ nrn2421

17. Vahdati Hassani F, Naseri V, Razavi BM, Mehri S, Abnous K, Hosseinzadeh H. Antidepressant effects of crocin and its effects on transcript and protein levels of CREB, BDNF, and VGF in rat hippocampus. Daru. 2014;22(1):16-25. https://doi.org/10.1186/2008-2231-22-16 
18. Dastgerdi AH, Radahmadi M, Pourshanazari AA, Dastgerdi HH. Effects of crocin on learning and memory in rats under chronic restraint stress with special focus on the hippocampal and frontal cortex corticosterone levels. Adv Biomed Res. 2017;6(1):157-168. https://doi.org/10.4103/abr. abr_107_17

19. Lopresti AL, Drummond PD. Saffron (Crocus sativus) for depression: a systematic review of clinical studies and examination of underlying antidepressant mechanisms of action. Hum Psychopharmacol. 2014;29(6):517-527. https://doi.org/10.1002/hup.2434

20. García-Rojo G, Fresno C, Vilches N, DíazVéliz G, Mora S, Aguayo F, et al. The ROCK inhibitor fasudil prevents chronic restraint stress-induced depressive-like behaviors and dendritic spine loss in rat hippocampus. Int $J$ Neuropsychopharmacol. 2017;20(4):336-345. https://doi.org/10.1093/ijnp/pyw108

21. Lee B, Sur B, Park J, Kim S-H, Kwon S, Yeom $\mathrm{M}$, et al. Chronic administration of baicalein decreases depression-like behavior induced by repeated restraint stress in rats. Korean $J$ Physiol Pharmacol. 2013;17(5):393-403. https://doi.org/10.4196/kjpp.2013.17.5·393

22. Lee B, Shim I, Lee H, Hahm D-H. Fucoidan prevents depression-like behavior in rats exposed to repeated restraint stress. $J$ Nat Med. 2013;67(3):534-544. https://doi.org/10.1007/ s11418-012-0712-5

23. Drossopoulou G, Antoniou K, Kitraki E, Papathanasiou G, Papalexi E, Dalla C, et al. Sex differences in behavioral, neurochemical and neuroendocrine effects induced by the forced swim test in rats. Neuroscience. 2004;126(4):849-857. https://doi.org/10.1016/ j.neuroscience.2004.04.044

24. Kalantarzadeh E, Radahmadi M, Reisi P. Effects of different dark chocolate diets on memory functions and brain corticosterone levels in rats under chronic stress. Physiol Pharmacol. 2020;24(3):185-196. https://doi.org/10.32598/ ppj.24.3.40

25. Dastgerdi HH, Radahmadi M, Reisi P, Dastgerdi AH. Effect of Crocin, exercise, and crocinaccompanied exercise on learning and memory in rats under chronic unpredictable stress. $A d v$ Biomed Res. 2018;7(1):137-147. https://doi.org/ 10.4103/abr.abr_153_18
26. Radahmadi M, Alaei H, Sharifi M-R, Hosseini $\mathrm{N}$. The effect of synchronized forced running with chronic stress on short, mid and longterm memory in rats. Asian $J$ Sports Med. 2013;4(1):54-62. https://doi.org/10.5812/asjsm .34532

27. Shabani M, Divsalar K, Janahmadi M. Destructive effects of prenatal WIN 55212-2 exposure on central nervous system of neonatal rats. Addict Health. 2012;4(1-2):9-19.

28. Vohora D, Pal S, Pillai K. Effect of locomotor activity on the passive avoidance test for the evaluation of cognitive function. Indian $J$ Pharmacol. 2000;32(3):242-245.

29. Hui J, Zhang J, Pu M, Zhou X, Dong L, Mao $\mathrm{X}$, et al. Modulation of GSK- $3 \beta / \beta$-catenin signaling contributes to learning and memory impairment in a rat model of depression. Int $J$ Neuropsychopharmacol. 2018;21(9):858-870. https://doi.org/10.1093/ijnp/pyyo40

30. Darcet F, Mendez-David I, Tritschler L, Gardier AM, Guilloux J-P, David DJ. Learning and memory impairments in a neuroendocrine mouse model of anxiety/depression. Front Behav Neurosci. 2014;8:136-149. https://doi. org/10.3389/fnbeh.2014.00136

31. Gao LC, Wang YT, Lao X, Wang C, Wang FY, Yuan CG. The change of learning and memory ability in the rat model of depression. Fen $\mathrm{Zi} \mathrm{Xi}$ Bao Sheng Wu Xue Bao. 2009;42(1):20-26.

32. Bhagya V, Srikumar B, Raju T, Rao BS. Chronic escitalopram treatment restores spatial learning, monoamine levels, and hippocampal long-term potentiation in an animal model of depression. Psychopharmacology (Berl). 2011;214(2):477494. https://doi.org/10.1007/s00213-010-2054-X

33. Ceglia I, Acconcia S, Fracasso C, Colovic M, Caccia S, Invernizzi RJB. Effects of chronic treatment with escitalopram or citalopram on extracellular 5 -HT in the prefrontal cortex of rats: role of 5 -HT1A receptors. $\mathrm{Br} J$ Pharmacol. 2004;142(3):469-478. https://doi.org/10.1038/ sj.bjp.0705800 
34. Soczynska JK, Ravindran LN, Styra R, McIntyre RS, Cyriac A, Manierka MS, et al. The effect of bupropion XL and escitalopram on memory and functional outcomes in adults with major depressive disorder: results from a randomized controlled trial. Psychiatry Res. 2014;22o(12):245-250. https://doi.org/10.1016/j.psychres .2014 .06 .053

35. Pitsikas N, Zisopoulou S, Tarantilis PA, Kanakis CD, Polissiou MG, Sakellaridis N. Effects of the active constituents of Crocus sativus L., crocins on recognition and spatial rats' memory. Behav Brain Res. 2007;183(2):141-146. https://doi.org/10.1016/j.bbr.2007.06.001

36. Hadipour M, Kaka G, Bahrami F, Meftahi GH, Pirzad Jahromi G, Mohammadi A, et al. Crocin improved amyloid beta induced long-term potentiation and memory deficits in the hippocampal CA1 neurons in freely moving rats. Synapse. 2018;72(5):e22026. https://doi.org/10.1002/syn.22026

37. Skandali N, Rowe JB, Voon V, Deakin JB, Cardinal RN, Cormack F, et al. Dissociable effects of acute SSRI (escitalopram) on executive, learning and emotional functions in healthy humans. Neuropsychopharmacology. 2018;43(13):2645-2651. https://doi.org/10 $.1038 / \mathrm{s} 41386-018-0229-\mathrm{z}$

38. Gaddy MA, Ingram RE. A meta-analytic review of mood-congruent implicit memory in depressed mood. Clin Psychol Rev. 2014;34(5):402-416. https://doi.org/10.1016/j.cpr.2014.06.001

39. McClintock SM, Husain MM, Greer TL, Cullum CM. Association between depression severity and neurocognitive function in major depressive disorder: a review and synthesis. Neuropsychology. 2010;24(1):9-34. https://doi .org/10.1037/aoo17336

40. Fossati P, Harvey P-O, Le Bastard G, Ergis A-M, Jouvent R, Allilaire J-F. Verbal memory performance of patients with a first depressive episode and patients with unipolar and bipolar recurrent depression. $J$ Psychiatr Res. 2004;38(2):137-144. https://doi.org/10.1016/ j.jpsychires.2003.08.002

41. Harmer CJ, Bhagwagar Z, Cowen PJ, Goodwin GM. Acute administration of citalopram facilitates memory consolidation in healthy volunteers. Psychopharmacology (Berl). 2002;163(1):106110. https://doi.org/10.1007/s00213-002-1151-x
42. Jensen JB, du Jardin KG, Song D, Budac D, Smagin G, Sanchez C, et al. Vortioxetine, but not escitalopram or duloxetine, reverses memory impairment induced by central 5 -HT depletion in rats: evidence for direct 5 -HT receptor modulation. Eur Neuropsychopharmacol. 2014;24(1):148-159. https://doi.org/10.1016/ j.euroneuro.2013.10.011

43. Rose EJ, Simonotto E, Spencer EP, Ebmeier KP. The effects of escitalopram on working memory and brain activity in healthy adults during performance of the $n$-back task. Psychopharmacology (Berl). 2006;185(3):339347. https://doi.org/10.1007/s00213-006-0334-2

44. McLaughlin KJ, Gomez JL, Baran SE, Conrad CD. The effects of chronic stress on hippocampal morphology and function: an evaluation of chronic restraint paradigms. Brain Res. 2007;1161:56-64. https://doi.org/10.1016/j. brainres.2007.05.042

45. Adabizadeh M, Mehri S, Rajabpour M, Abnous $\mathrm{K}$, Rashedinia M, Hosseinzadeh $\mathrm{H}$. The effects of crocin on spatial memory impairment induced by hyoscine: role of NMDA, AMPA, ERK and CaMKII proteins in rat hippocampus. Iran $J$ Basic Med Sci. 2019;22(6):601-609. https://doi.org/10.22038/ijbms.2019.30138.7266

46. Hosseinzadeh H, Sadeghnia HR, Ghaeni FA, Motamedshariaty VS, Mohajeri SA. Effects of saffron (Crocus sativus L.) and its active constituent, crocin, on recognition and spatial memory after chronic cerebral hypoperfusion in rats. Phytother Res. 2012;26(3):381-386. https://doi.org/10.1002/ptr.3566

47. Tamaddonfard E, Farshid AA, Asri-Rezaee S, Javadi S, Khosravi V, Rahman B, et al. Crocin improved learning and memory impairments in streptozotocin-induced diabetic rats. Iran J Basic Med Sci. 2013;16(1):91-100.

48. Heidari S, Mehri S, Hosseinzadeh H. Memory enhancement and protective effects of crocin against D-galactose aging model in the hippocampus of Wistar rats. Iran $J$ Basic Med Sci. 2017;20(11):1250-1259. https://doi.org/10 $.22038 /$ IJBMS.2017.9541 
49. Ahmadi M, Rajaei Z, Hadjzadeh M, Nemati H, Hosseini M. Crocin improves spatial learning and memory deficits in the Morris water maze via attenuating cortical oxidative damage in diabetic rats. Neurosci Lett. 2017;642:1-6. https://doi.org/10.1016/j.neulet.2017.01.049

50. Alboni S, Benatti C, Capone G, Corsini D, Caggia F, Tascedda F, et al. Time-dependent effects of escitalopram on brain derived neurotrophic factor (BDNF) and neuroplasticity related targets in the central nervous system of rats. Eur $J$ Pharmacol. 2010;643(2-3):180-187. https://doi.org/10.1016/j.ejphar.2010.06.028

51. Dorri SA, Hosseinzadeh H, Abnous K, Hasani FV, Robati RY, Razavi BM. Involvement of brain-derived neurotrophic factor (BDNF) on malathion induced depressive-like behavior in subacute exposure and protective effects of crocin. Iran J Basic Med Sci. 2015;18(10):958-966.

52. Khalatbari-Mohseni A, Banafshe HR, Mirhosseini $\mathrm{N}$, Asemi Z, Ghaderi A, Omidi A. The effects of crocin on psychological parameters in patients under methadone maintenance treatment: a randomized clinical trial. Subst Abuse Treat Prev Policy. 2019;14(1):9-17. https://doi.org/10 $.1186 / \mathrm{s} 13011-019-0198-1$

53. Talaei A, Moghadam MH, Tabassi SAS, Mohajeri SA. Crocin, the main active saffron constituent, as an adjunctive treatment in major depressive disorder: a randomized, doubleblind, placebo-controlled, pilot clinical trial. $J$ Affect Disord. 2015;174:51-56. https://doi.org/ 10.1016/j.jad.2014.11.035

54. Zhang L, Previn R, Lu L, Liao R-F, Jin Y, Wang R-K. Crocin, a natural product attenuates lipopolysaccharide-induced anxiety and depressive-like behaviors through suppressing NF-kB and NLRP3 signaling pathway. Brain Res Bull. 2018;142:352-359. https://doi.org/ 10.1016/j.brainresbull.2018.08.021

55. Shalaby A, Kamal S. Effect of escitalopram on GABA level and anti-oxidant markers in prefrontal cortex and nucleus accumbens of chronic mild stress-exposed albino rats. Int $J$ Physiol Pathophysiol Pharmacol. 2009;1(2): 154-161.
56. Soeda S, Ochiai T, Paopong L, Tanaka H, Shoyama Y, Shimeno H. Crocin suppresses tumor necrosis factor- $\alpha$-induced cell death of neuronally differentiated PC-12 cells. Life Sci. 2001;69(24):2887-2898. https://doi.org/10 .1016/soo24-3205(01)01357-1

57. Powell TR, Schalkwyk LC, Heffernan AL, Breen G, Lawrence T, Price T, et al. Tumor necrosis factor and its targets in the inflammatory cytokine pathway are identified as putative transcriptomic biomarkers for escitalopram response. Eur Neuropsychopharmacol. 2013;23(9):1105-1114. https://doi.org/10.1016/j.euroneuro.2012.09.009

58. Ghalandari-Shamami M, Nourizade S, Yousefi B, Vafaei AA, Pakdel R, Rashidy-Pour A. beneficial effects of physical activity and crocin against adolescent stress induced anxiety or depressivelike symptoms and dendritic morphology remodeling in prefrontal cortex in adult male rats. Neurochem Res. 2019;44(4):917-929. https://doi.org/10.1007/s11064-019-02727-2

59. Benatti C, Alboni S, Blom JM, Mendlewicz J, Tascedda F, Brunello N. Molecular changes associated with escitalopram response in a stress-based model of depression. Psychoneuroendocrinology. 2018;87:74-82. https://doi.org/10.1016/j.psyneuen.2017.10.011

60. Hansson AC, Rimondini R, Heilig M, Mathé AA, Sommer WH. Dissociation of antidepressantlike activity of escitalopram and nortriptyline on behaviour and hippocampal BDNF expression in female rats. $J$ Psychopharmacol. 2011;25(10):1378-1387. https://doi.org/10.1177/ 0269881110393049

61. Matrisciano F, Bonaccorso S, Ricciardi A, Scaccianoce S, Panaccione I, Wang L, et al. Changes in BDNF serum levels in patients with major depression disorder (MDD) after 6 months treatment with sertraline, escitalopram, or venlafaxine. $J$ Psychiatr Res. 2009;43(3):247-254. https://doi.org/10.1016/j. jpsychires.2008.03.014

62. Yang J, Pei Y, Pan Y-L, Jia J, Shi C, Yu Y, et al. Enhanced Antidepressant-Like Effects of Electroacupuncture Combined with Citalopram in a Rat Model of Depression. Evid Based Complement Alternat Med. 2013;2013:1-12. https://doi.org/10.1155/2013/107380 
63. Ge J-F, Qi C-C, Zhou J-N. Imbalance of leptin pathway and hypothalamus synaptic plasticity markers are associated with stressinduced depression in rats. Behav Brain Res. 2013;249:38-43. https://doi.org/10.1016/j.bbr .2013 .04 .020

64. Jarosik J, Legutko B, Unsicker K, und Halbach OvB. Antidepressant-mediated reversal of abnormal behavior and neurodegeneration in mice following olfactory bulbectomy. Exp Neurol. 2007;204(1):20-28. https://doi.org/10.1016/j. expneurol.2006.09.008

65. Hosseinzadeh H, Noraei NB. Anxiolytic and hypnotic effect of Crocus sativus aqueous extract and its constituents, crocin and safranal, in mice. Phytother Res. 2009;23(6):768-774. https://doi.org/10.1002/ptr.2597
66. Amin B, Nakhsaz A, Hosseinzadeh H. Evaluation of the antidepressant-like effects of acute and sub-acute administration of crocin and crocetin in mice. Avicenna $J$ Phytomed. 2015;5(5):458468.

67. Lin DH, Zhang XR, Ye DQ, Xi GJ, Hui JJ, Liu SS, et al. The role of the two-pore domain potassium channel TREK-1 in the therapeutic effects of escitalopram in a rat model of poststroke depression. CNS Neurosci Ther. 2015;21(6): 504-512. https://doi.org/10.1111/cns.12384

68. Prinssen EP, Ballard TM, Kolb Y, Nicolas LB. The effects of serotonin reuptake inhibitors on locomotor activity in gerbils. Pharmacol Biochem Behav. 2006;85(1):44-49. https://doi. org/10.1016/j.pbb.2006.07.005 